\section{Tratamiento comunitario para el control del tracoma}

Según un estudio que se publicó en el número del 21 de agosto de la revista The Lancet, la administración a toda una comunidad de un tratamiento corto con el antibiótico oral azitromicina se ha mostrado más eficaz para el control del tracoma que el tratamiento estándar, que suele consistir en la aplicación diaria de ungüento de tetraciclina durante 6 semanas. Se ha sabido desde hace mucho tiempo que existían los antibióticos para tratar esta enfermedad, que es una de las principales causas de ceguera en el mundo, pero hasta hace poco no se disponía de una vía de administración apropiada.

Cerca de 600 millones de personas viven en zonas donde el tracoma es endémico; de ellas, 150 millones padecen de enfermedad activa. Los niños son especialmente susceptibles al tracoma, que obedece a la infección de la superficie interna del párpado por la bacteria Chlamydia trachomatis. Después de episodios recurrentes de conjuntivitis, que se inician en la niñez, se forma un tejido cicatrizal que lleva a la inversión del párpado y a lesiones secundarias de la córnea. En comunidades donde el tracoma es endémico, $25 \%$ de las personas entre los 50 y 60 años de edad pueden volverse ciegas. Estas personas muchas veces no pueden ganarse la vida y se convierten en una carga económica para su familia y la sociedad.

Actualmente, alrededor de seis millones de personas están ciegas o gravemente discapacitadas como resultado de la infeción ocular. El tratamiento con azitromicina, según explica el Director del Instituto Nacional de Alergias y Enfermedades Crónicas de los Estados Unidos de América, provee beneficios a largo plazo que no suelen estar al alcance de personas en países en desarrollo. El estudio publicado en The Lancet, llevado a cabo por investigadores de la Universidad de California en Los Ángeles, demostró la superioridad de un tratamiento a base de tres dosis de azitromicina oral a intervalos semanales, al compararse con el régimen estándar a base de tetraciclina tópica. Concluyeron los autores del estudio que debido a su eficacia y a la facilidad de su administración, la azitromicina es un componente importante de los programas de control del tracoma. Aunque el medicamento es más caro que el ungüento de tetraciclina, la carestía puede verse compensada por el mayor costo de distribuir y administrar varias dosis del ungüento.

El tratamiento con antibióticos es uno de los componentes de la estrategia para el control del tracoma denominada SAFE: consiste en cirugía (surgery) en casos avanzados; antibióticos (antibiotics) para tratar y prevenir las infecciones; lavado de la cara (face washing) y buena higiene personal; y mejoras ambientales (environmental improvement), tales como un mejor acceso a agua limpia y a saneamiento y educación sanitaria. Esta estrategia, que incluye el tratamiento con azitromicina, puede aportar grandes beneficios a personas que habitan en zonas endémicas porque reduce las infecciones individuales y la infección persistente en zonas con baja prevalencia.

El régimen tradicional a base de ungüento de tetraciclina solamente trata la infección ocular y su eficacia es de corta duración. Por otra parte, los antibióticos orales de acción corta también pueden usarse, pero la necesidad de administrar dosis múltiples los torna menos eficaces en comparación con menos dosis de un antibiótico oral de acción más prolongada. El régimen oral con azitromicina se acompaña de mejor adhesión por parte de los pacientes y resulta promisorio para el control del tracoma cuando se aplica a comunidades enteras. (National Institute of Allergy and Infectious Diseases. A leading cause of blindness may be controlled by simple course of oral antibiotic. Comunicado de prensa, 19 de agosto de 1999).

\section{Razón cintura:caderas como factor predictor de hipertensión arterial}

En 1985, la Conferencia sobre las Implicaciones de la Obesidad para la Salud señaló que la gordura corporal y su topografía ayudan a predecir la ulterior aparición de enfermedades crónicas, tales como cardiopatía coronaria, cáncer, hiperlipidemias, diabetes del adulto, y otras. Asimismo, los resultados de numerosos estudios apuntan a una estrecha relación entre un exceso de tejido graso en la región abdominal y el infarto del miocardio, los accidentes cerebrovasculares y la diabetes. La OMS recomienda usar la antropometría para vigilar los factores de riesgo de las enfermedades crónicas y realizar estudios para definir la asociación entre di- 
chos factores y resultados específicos, como la hipertensión arterial.

En la Universidad Federal de Rio de Janeiro, Brasil, un grupo de investigadores ha estudiado la asociación entre la razón cintura:caderas (RCC) y la tensión arterial. El estudio, que se basó en un muestreo bietápico por conglomerados, se efectuó en 3282 individuos mayores de 20 años que fueron encuestados en Rio de Janeiro en 1995-1996. A cada individuo se le midieron la estatura, el peso, la circunferencia de la cintura y de las caderas y la tensión arterial. Se consideró hipertensa a toda persona con una tensión sistólica $\geq 140 \mathrm{mmHg}$ o diastólica $\geq 90 \mathrm{mmHg}$, o en tratamiento con antihipertensivos. Se calcularon la sensibilidad y especificidad de distintos puntos de corte de la RCC para predecir la hipertensión arterial según edad, sexo y presencia de sobrepeso, usando como base los parámetros de la OMS.

El estudio no reveló diferencias importantes en la capacidad predictiva de la RCC en distintas edades, pero sí mostró la necesidad de tener diferentes puntos de corte para hombres y mujeres. Se encontró que el mejor punto de corte para los hombres era de 0,95 y para las mujeres, de 0,80 . En comparación con la razón cintura:estatura (RCE) y la circunferencia de la cintura (CC), la RCC tuvo mayor capacidad para predecir la hipertensión arterial y mostró menor correlación con el índice de masa corporal, lo cual implica que una RCC alta obedece principalmente a la concentración de la gordura en la región abdominal y no a un aumento de la masa corporal total. Esto a su vez permite identificar con mayor precisión a las personas en riesgo de sufrir enfermedades crónicas, particularmente la hipertensión arterial. Al compararse entre sí los diferentes valores antropométricos, la RCE mostró la especificidad más baja; la RCC mostró el mejor rendimiento entre los hombres; en las mujeres, la CC y la RCC se comportan de forma similar $\mathrm{y}$, en mujeres con exceso de peso, la CC tiene alta sensibilidad y baja especificidad.

La distribución de la gordura en los diferentes compartimientos del cuerpo sigue siendo objeto de estudio, ya que se cree que la presencia de tejido graso cerca de las vísceras, más que en el panículo adiposo subcutáneo, es factor de riesgo de morbilidad y mortalidad. Para cuantificar la grasa visceral es necesario aplicar tecnologías de alto costo, como la tomografía computadorizada o la resonancia magnética. Se usan, por lo tanto, diferentes medidas antropométricas como marcadores de esa distribución. (Pereira AR, Sichieri R, Marins MRV. Razao cintura/quadril como preditor de hipertensão arterial. Cad Saude Publica 1999;15(2):333-344).

\section{El cinc y su efecto sobre la duración de la diarrea persistente}

La diarrea crónica o persistente (de más de 14 días de duración) en niños se asocia con un aumento de la susceptibilidad a las infecciones y con un retraso del crecimiento. En países como el Perú, la mitad de las defunciones asociadas con episodios de diarrea ocurren en niños con diarrea persistente. Aunque algunos agentes patógenos específicos están vinculados con este tipo de diarrea, estudios microbiológicos de muestras fecales secuenciales han revelado que en algunos casos la cronicidad se debe a una serie de infecciones nuevas que se producen antes de que el niño se haya recuperado por completo de la infección anterior. Las posibilidades de que esto ocurra se ven aumentadas en ambientes domésticos caracterizados por poca higiene y un alto grado de contaminación fecal. También contribuyen algunos factores inmunológicos y ciertas deficiencias nutricionales del huésped que aumentan su susceptibilidad a las infecciones entéricas y retrasan la recuperación de las mucosas.

El cinc es un micronutriente esencial para muchas funciones celulares, entre ellas la transcripción del ácido desoxirribonucleico (ADN) y la mitosis. Por lo tanto no es de sorpreder que la deficiencia de cinc, reflejada en una reducción de sus concentraciones plasmáticas, se asocie con un riesgo aumentado de diarrea y con episodios diarreicos de mayor gravedad. Por otra parte, se ha demostrado que la administración de suplementos de cinc facilitan la recuperación de la mucosa intestinal.

La deficiencia de cinc suele ser común en poblaciones de países pobres debido al poco consumo de carnes ricas en este nutriente y a la baja biodisponibilidad del mismo a partir de los productos de origen vegetal que integran la dieta habitual. En las afueras de Lima, Perú, un grupo de investigadores llevó a cabo un estudio aleatorio a doble ciego en una de las comunidades más pobres de la ciudad, donde los domicilios carecen de alcantarillado y de un sistema formal para la eliminación de excreta. A los niños de 6 a 36 meses de edad con diarrea persistente se les administró diariamente durante 2 semanas placebo (grupo $\mathrm{P}, n=136$ ) o un suplemento de $20 \mathrm{mg}$ de cinc, con (grupo $\mathrm{Z}+\mathrm{VM}, n=137$ ) o sin (grupo $Z, n=139$ ) vitaminas y minerales adicionales. Se hizo un seguimiento diario de todo síntoma de enfermedad y se llevaron a cabo evaluaciones bioquímicas y antropométricas los días primero y decimoquinto del estudio.

Los dos grupos que recibieron un tratamiento tuvieron valores de base similares en cuanto a las características del primer episodio, datos antropo- 
métricos y concentración de cinc en plasma. Los niños consumieron un promedio de $95 \%$ (grupo $\mathrm{P}$ ), 94\% (grupo Z) u 88\% (grupo Z + VM) del suplemento $(P<0,001)$. La concentración plasmática de cinc no varió de forma significativa entre el primer día y el decimoquinto en el grupo $\mathrm{P}(4 \mu \mathrm{g} / \mathrm{dL})$, pero aumentó $38 \mu \mathrm{g} / \mathrm{dL}$ en el grupo $\mathrm{Z}$ y $14 \mu \mathrm{g} / \mathrm{dL}$ en el grupo $\mathrm{Z}+\mathrm{VM}$. La duración media de la diarrea después de iniciarse el tratamiento fue de 1 día; en los niños que siguieron teniendo diarrea, el tratamiento tuvo un efecto notable en la duración del cuadro clínico $(P=0,04$, análisis de la covarianza). En particular, la duración de la enfermedad se redujo en $28 \%$, valor con significación estadística, en niños del grupo $Z(P=0,01)$ y en $33 \%$ en niñas del grupo $Z+V M(P=0,04)$. No se observó ninguna diferencia en la gravedad del episodio en los distintos grupos. En resumen, se produjo una reducción significativa de la duración de la diarrea persistente en subgrupos seleccionados de niños peruanos tratados ambulatoriamente con suplementos de cinc. (Penny ME, Peerson JM, Marin RM, Duran A, Lanata CF, Lönnerdal B, Black RE, Brown KH. Randomized, community-based trial of the effect of zinc supplementation, with and without other micronutrients, on the duration of persistent childhood diarrhea in Lima, Peru. J Pediatr 1999;135:208-217).

\section{La administración de ácido fólico a embarazadas para prevenir defectos del tubo neural}

Actualmente se acepta sin ningún lugar a dudas que el ácido fólico administrado a mujeres embarazadas confiere protección contra defectos del tubo neural (DTN) en neonatos. Como resultado de ello, casi todos los países tienen recomendaciones casi idénticas al respecto: 4 a 5 mg de ácido fólico al día por la vía oral para prevenir la recurrencia de DTN. No obstante, 95\% de los casos de DTN son el primero en los hijos de una misma mujer y evitar estos primeros casos representa un problema mayor que el de prevenir las recurrencias. Para prevenir un primer caso de DTN se recomienda que la madre ingiera un suplemento diario de $400 \mu \mathrm{g}$ de ácido fólico, cantidad que representa tres veces más que la ingesta promedio habitual y que es difícil de alcanzar en condiciones normales. Se han propuesto tres fuentes distintas de ácido fólico adicional: a) un aumento del consumo de alimentos ricos en esta vitamina; b) el consumo de suplementos de ácido fólico; y c) fortificación de los alimentos con ácido fólico. Algunos estudios han revelado, sin embargo, que un mayor consumo de alimentos ricos en esta vitamina no tiene la misma eficacia que un consumo equivalente en forma de productos fortificados con ella. Los suplementos pueden aumentar mucho las concentraciones de folato en el plasma y los glóbulos rojos de mujeres que los han recibido en forma experimental, pero en la población en general no es fácil conseguir una buena adhesión al régimen diario de suplementación.

La fortificación con ácido fólico de los productos a base de granos se hizo obligatoria hace muy poco tiempo en los Estados Unidos de América, con la expectativa de poder aumentar el consumo total de folatos en $100 \mu \mathrm{g}$ al día. Para pronosticar la eficacia de esta medida, un grupo de investigadores efectuó un estudio en el que se eliminó una cantidad equivalente de alimentos fortificados con folato de la dieta de mujeres jóvenes que habían sido expuestas por elección a fortificación crónica con ácido fólico. La medida tuvo por objetivo determinar el efecto en la cantidad de folato en el organismo del consumo de alimentos con poca fortificación.

Se cuantificó la diferencia en la ingesta diaria de folatos y se midió la concentración de folatos en glóbulos rojos y en el plasma de mujeres que habían suspendido la ingestión de alimentos fortificados con folatos por un período de 12 semanas, tras haber tenido en su dieta habitual una ingestión semanal de por lo menos un alimento fortificado. Las personas que siguieron consumiendo alimentos ricos en folatos por lo menos una vez a la semana $(n=21)$ tuvieron un consumo total de folatos más alto $(P=0,002)$ que las que los consumieron menos de una vez a la semana $(n=30)$ y también mostraron concentraciones de folato en glóbulos rojos más altas $(P=0,023)$ que estas. Asimismo, la exclusión durante 12 semanas de alimentos ricos en folatos dio por resultado una disminución de la ingestión de folatos de $78 \pm 56 \mu$ g diarios $(P<0,001)$, que se manifestó en una reducción estadísticamente significativa de $111 \mathrm{nmol} / \mathrm{L}(49 \mu \mathrm{g} / \mathrm{L})$ en las concentraciones de folatos en los glóbulos rojos $(P<0,05)$.

Se puede concluir a partir de estos resultados que si, a la inversa, se aumenta en $78 \mu \mathrm{g}$ diarios el consumo de folatos como resultado de la nueva fortificación obligatoria de los alimentos en los Estados Unidos, se obtendrá un aumento equivalente al observado en este estudio de las concentraciones hemáticas de esta vitamina. Ello a su vez tendrá un efecto favorable, aunque no óptimo, en la prevención de defectos del tubo neural. (Cuskelly GJ, McNulty H, Scott JM. Fortification with low amounts of folic acid makes a significant difference in folate status in young women: implications for the prevention of neural tube defects. Am J Clin Nutr 1999;70:234-239). 


\section{La contaminación ambiental en tres grandes urbes latinoamericanas}

Aunque el crecimiento urbano rápido y desordenado y la industrialización hoy en día dan lugar a la acumulación de riesgos para la salud debido a la contaminación atmosférica que con ellos se asocia, son escasos los programas destinados a proteger el medio ambiente, particularmente el aire. En los países latinoamericanos, el proceso de urbanización ha sido muy acelerado durante todo el siglo XX. Más de 14 aglomeraciones urbanas tienen en exceso de dos millones de habitantes. De ellas, São Paulo, Brasil, y México, D.F. se encuentran entre las 12 ciudades más grandes del mundo. En 1995, ambas ciudades presentaban tasas de crecimiento muy elevadas y tenían alrededor de $76 \%$ del total de la población. Ese mismo año Santiago, Chile, presentaba una tasa anual de crecimiento urbano menor de $1,8 \%$, pero su población representaba $84 \%$ de la población total del país. En un artículo reciente se examinó la problemática ambiental en estas tres megaciudades de la Región y se repasaron los programas para el control de la contaminación atmosférica que han puesto en marcha sus respectivos gobiernos, así como también el impacto de dichos programas en la evolución de las concentraciones de sustancias contaminantes de 1988 a 1995 en Santiago, Chile, y São Paulo y hasta 1997 en México, D.F.

En todas las ciudades se observó un descenso de las concentraciones de partículas totales en suspensión (PTS), partículas de diámetro menor de $10 \mu \mathrm{m}\left(\mathrm{PM}_{10}\right)$, bióxido de azufre $\left(\mathrm{SO}_{2}\right)$, bióxido de nitrógeno $\left(\mathrm{NO}_{2}\right)$, monóxido de carbono $(\mathrm{CO})$ y ozono $\left(\mathrm{O}_{3}\right)$ durante el período mencionado, aunque en su mayor parte estas sustancias contaminantes siguen rebasando la norma de calidad. Esto últmo podría deberse, en parte, al aumento del parque vehicular. Los tres programas urbanos han tenido su mayor impacto en las concentraciones de $\mathrm{SO}_{2}$ habiéndose logrado con ellos reducir estas concentraciones a valores inferiores al establecido como norma por la Agencia de Protección Ambiental (EPA) de los Estados Unidos de América (promedio annual de $30 \mathrm{ppb}$ ). La reducción se ha obtenido principalmente como resultado del control de la emisión de azufre por fuentes fijas y de la disminución del contenido de azufre en el diesel, tratándose en ambos casos de medidas más baratas que las necesarias para reducir otros contaminantes atmosféricos. La concentración elevada de $\mathrm{O}_{3}$ sigue siendo un problema importante en las tres ciudades y par- ticularmente en México, D.F., donde suele rebasar la norma de la EPA más de $90 \%$ del tiempo.

Las concentraciones persistentemente altas de partículas ambientales, que ponen a la población en riesgo de morbilidad y mortalidad prematuras, hacen necesario establecer nuevas medidas de control. Según estudios recientes, la fracción más importante desde el punto de vista de su efecto nocivo en la salud es la de partículas finas procedentes de la combustión $\left(\mathrm{PM}_{25}\right)$ y quizá la de partículas ultrafinas (que contienen nitratos, sulfatos, ácidos fuertes y elementos traza, tales como los metales de transición). Las partículas finas contienen una mayor superficie de agregación que las gruesas $\left(\mathrm{PM}_{10-25}\right)$, lo cual facilita la adsorción de componentes potencialmente tóxicos y la disolución o absorción de contaminantes gaseosos y su deposición en la región torácica. Debido a esta situación, la EPA ha propuesto revisar las normas actuales con respecto a la carga de partículas ambientales, agregando dos valores nuevos como límites de concentración para partículas finas: media anual de $15 \mu \mathrm{g} / \mathrm{m}^{3}$ y promedio de 24 horas de 50 $\mu \mathrm{g} / \mathrm{m}^{3}$. Esto tiene por objeto incrementar la protección contra los efectos nocivos de las partículas en suspensión, que abarcan mortalidad prematura; aumento de las admisiones a hospitales; aumento de síntomas respiratorios; alteración de la función pulmonar y de los mecanismos de defensa respiratorios, entre otros.

Se torna necesario considerar otras medidas para el control de la contaminación atmosférica que permitan reducir las emisiones tóxicas de los vehículos de motor, tales como el uso de combustibles limpios, la reducción del número de vehículos particulares, el mejoramiento del transporte público y la prohibición de la circulación de vehículos en las zonas céntricas de las grandes ciudades. Un componente de los programas para mejorar el medio ambiente debe consistir en políticas de transporte sustentable, que deben adoptarse con la mutua colaboración de industriales, políticos y proveedores de servicios de salud, pero sobre todo, con la participación de la comunidad. Es importante que en este sentido cada país de América Latina examine su situación particular a fin de encontrar las opciones de control más adecuadas para sus problemas de contaminación. (Lacasaña-Navarro M, AguilarGarduño C, Romieu I. Evolución de la contaminación del aire e impacto de los programas de control en tres megaciudades de América Latina. Salud Pu blica Mex 1999;41(3):203-215). 